\title{
Manipulating proteostasis to repair the F508del-CFTR defect in cystic fibrosis
}

\author{
Speranza Esposito ${ }^{1}$, Antonella Tosco ${ }^{2}$, Valeria R. Villella ${ }^{1}$, Valeria Raia ${ }^{2^{*}}$, Guido Kroemer ${ }^{3,4,5,6,7,8,9^{*}}$ and Luigi Maiuri ${ }^{1}, 10^{*}$
}

\begin{abstract}
Cystic fibrosis (CF) is a lethal monogenic disease caused by mutations in the cystic fibrosis transmembrane conductance regulator (CFTR) gene that entails the (diagnostic) increase in sweat electrolyte concentrations, progressive lung disease with chronic inflammation and recurrent bacterial infections, pancreatic insufficiency, and male infertility. Therapies aimed at restoring the CFTR defect have emerged. Thus, a small molecule which facilitates chloride channel opening, the potentiator Ivacaftor, has been approved for the treatment of CF patients bearing a particular class of rare CFTR mutations. However, small molecules that directly target the most common misfolded CFTR mutant, F508del, and improve its intracellular trafficking in vitro, have been less effective than expected when tested in CF patients, even in combination with Ivacaftor. Thus, new strategies are required to circumvent the F508del-CFTR defect. Airway and intestinal epithelial cells from CF patients bearing the F508del-CFTR mutation exhibit an impressive derangement of cellular proteostasis, with oxidative stress, overactivation of the tissue transglutaminase (TG2), and disabled autophagy. Proteostasis regulators such as cysteamine can rescue and stabilize a functional F508del-CFTR protein through suppressing TG2 activation and restoring autophagy in vivo in F508del-CFTR homozygous mice, in vitro in CF patientderived cell lines, ex vivo in freshly collected primary patient's nasal cells, as well as in a pilot clinical trial involving homozygous F508del-CFTR patients. Here, we discuss how the therapeutic normalization of defective proteostasis can be harnessed for the treatment of CF patients with the F508del-CFTR mutation.
\end{abstract}

Keywords: Autophagy, Proteostasis, Cystic fibrosis, CFTR, CFTR-repairing therapy

Cystic fibrosis (CF), the most common lethal monogenic disease in Caucasians, is caused by mutations in the gene coding for cystic fibrosis transmembrane conductance regulator (CFTR), a 1480-amino acid protein functioning as a chloride channel at the apical membrane from the epithelial cells [1]. CF is a systemic disease, although the extent of clinical manifestations is highly heterogeneous in distinct organs [1-3]. The phenotypic consequences of the defective CFTR function comprise insufficiency of the exocrine pancreas, increased electrolytes in sweat, male infertility due to congenital absence of the vas deferens, and-most prevalent-a debilitating progressive lung disease resulting from decreased

\footnotetext{
*Correspondence: raia@unina.it; kroemer@orange.fr; luigi.maiuri@gmail.com ${ }^{2}$ Regional Cystic Fibrosis Center, Pediatric Unit, Department of Translational Medical Sciences, Federico II University, Naples 80131, Italy

${ }^{3}$ Equipe 11 labellisée par la Ligue contre le Cancer, Centre de Recherche des Cordeliers, Paris, France

${ }^{1}$ European Institute for Research in Cystic Fibrosis, Division of Genetics and Cell Biology, San Raffaele Scientific Institute, Milan 20132, Italy

Full list of author information is available at the end of the article
}

mucociliary clearance with accumulation of thick, sticky mucus, chronic inflammation, and persistent and untreatable bacterial colonization causing frequent chest infections, mainly by Pseudomonas aeruginosa [1-3].

Exocrine pancreatic insufficiency frequently occurs in patients with CF. It is mainly associated with "severe" CFTR mutations, where both alleles are affected by complete or major loss of function. Other gastrointestinal complications comprise recurrent abdominal pain or acute recurrent pancreatitis. Moreover, thickened secretions blocked in the bile ducts may cause progressive liver damage. Salty-tasting skin, poor growth, and poor weight gain despite normal food intake often appear in infancy, as bowel obstruction due to meconium ileus may occur in neonates. The causes of growth failure are multifactorial and include chronic lung infection, poor digestibility and absorption of nutrients through the gastrointestinal tract, and increased metabolic demand due to chronic illness.

\section{Springer}

(c) 2016 Esposito et al. Open Access This article is distributed under the terms of the Creative Commons Attribution 4.0 International License (http://creativecommons.org/licenses/by/4.0/), which permits unrestricted use, distribution, and reproduction in any medium, provided you give appropriate credit to the original author(s) and the source, provide a link to the Creative Commons license, and indicate if changes were made. 
Diagnostic procedures in CF include routine newborn screening (when blood concentrations of trypsinogen are usually measured as a surrogate marker), sweat testing, and genetic analysis. Infants with an abnormal finding in newborn screen need the sweat test to confirm the CF diagnosis. In countries in which newborn screening is not available, most individuals are primarily diagnosed by means of the sweat test, in which pilocarpine is applied to the skin to stimulate local sweating followed by iontophoresis to determine the concentration of chloride. CF can also be diagnosed by the identification of mutations in the CFTR gene.

Despite increased survival to date, several treatment methods of CF are purely symptomatic and hence fail to address the primary cause of CF, namely the loss of function of CFTR. New anti-inflammatory and antibiotic drugs are on the agenda of drug discovery approaches and clinical trials in CF patients [4].

Approximately 2000 mutations, most of which are disease relevant, have been identified in the CFTR gene and then categorized in six different classes according to their functional impact [5]. They include severe CFTR mutations that result in negligible protein synthesis (class I), misfolded mutants with defective intracellular trafficking (class II), or mutated proteins that are orthotopically expressed but exhibit impaired channel function (class III). Mutation-specific approaches aimed at correcting the CFTR defect (CFTR-repairing therapies) have recently emerged $[5,6]$. These strategies are commonly focused on the identification of molecules that directly target mutant CFTR. These compounds are either capable of correcting the trafficking of CFTR mutants ("correctors": agents that ensure the expression of the mutated protein at the apical plasma membrane) or improving channel function ("potentiators": agents that reinstate the channel function of mutated CFTR proteins that are orthotopically expressed).

An orally available compound identified by highthroughput screening, the CFTR potentiator VX-770 (Ivacaftor, trade name Kalydeco), has been shown to efficiently reduce chloride levels in sweat and to improve lung function in CF patients harboring the G551D CFTR genotype, a rare class III CFTR mutant that affects only 4-5 \% of CF patients [7, 8]. However, no effective treatments are available for the most common class II CFTR mutations. Indeed, one single mutation, p.phe508delCFTR, commonly known as F508del-CFTR (categorized in class II), accounts for about $70 \%$ of CFTR chromosomes worldwide and is present in approximately $90 \%$ of CF patients [5, 6]. F508del-CFTR is a misfolded protein mostly retained at the endoplasmic reticulum where it is immediately sent for degradation [9]. Despite the gating defect linked to the impaired protein conformation, F508del-CFTR shows some degree of function if rescued and stabilized at the plasma membrane (PM) [3-5]. Restoring about $20-30 \%$ of CFTR function is believed to confer an at least partial clinical benefit to CF patients. However, although F508delCFTR can be rescued at the PM by CFTR correctors in vitro [9], this mutant CFTR protein is unstable at the cell surface and rapidly redirected from endosomal recycling towards lysosomal delivery and degradation. The investigational F508del corrector VX-809 (Lumacaftor), which is endowed with rescuing efficacy in vitro and in primary cultures of lung cells from F508del-CFTR homozygous CF patients [9], showed only the modest efficacy in a phase II clinical trial in CF patients homozygous for the F508del-CFTR mutant [10]. Moreover, only marginal effects on lung function have been observed in phase II, and phase III randomized clinical trials aimed at testing the efficacy of a combination of the corrector Lumacaftor and the potentiator Ivacaftor [11-14]. Notably, no effects of treatment on sweat chloride, a surrogate marker of CFTR function in vivo, have been reported [12]. New combinations of correctors and potentiators are being evaluated in clinical trials in $\mathrm{CF}$ patients bearing F508del-CFTR mutation. However, the preclinical evidence supporting the putative effectiveness of chronic administration of the potentiator VX-770 together with a CFTR corrector (either VX-809 or VX661) is lacking, and the mechanisms of action of these investigational correctors are still poorly understood. This suggests that interventions other than simple correction or potentiation of mutated CFTR are required in CF therapy.

\section{Proteostasis network is deranged in CF epithelia}

Recently, more general therapeutic strategies aiming at the improvement of proteostasis have emerged [15-18]. These strategies have been designed to improve the cellular environment perturbed by the lack of a functional CFTR instead of directly targeting the mutant CFTR protein. Such a novel approach is based on recent evidence indicating that CFTR does not act as a pure ion channel but is a platform for cellular signaling within a proper cellular environment. Given the heterogeneity of signaling pathways, this new vision of CFTR implies that its function is conditioned by its cellular environment. Importantly, the protein interactomes of wild-type (WT) CFTR and the most frequent CFTR mutant, F508delCFTR, are rather different $[17,18]$. There is growing consensus that indirect measures to correct the deficient proteostasis of CFTR may be fruitful. Proteostasis modulators may reestablish the plasma membrane localization and function of F508del-CFTR by remodeling the F508del-CFTR interactome, for instance by avoiding unwanted interactions and reinstating desirable proteinprotein interactions for F508del-CFTR [17]. Notably, 
CFTR is a key player of proteostasis in the epithelial cells, as the inhibition of CFTR function derails the intracellular environment and ignites the disposal of CFTR itself in a feed-forward loop [19].

A complex derangement of proteostasis takes place in human bronchial F508del-CFTR homozygous epithelial cell lines as well as in the lungs from F508del-CFTR homozygous $\left(C f t^{F 508 d e l}\right)$ mice [20-23]. Dysfunctional F508del-CFTR protein induces a complex alteration of the post-translational network with increased generation of reactive oxygen species (ROS) that lead to SUMOylation with decreased ubiquitylation and persistent activation of pro-fibrotic tissue transglutaminase 2 (TG2) [20, 21]. TG2 is a versatile multifunctional protein that changes its function depending on external and internal signals [24-26]. In the presence of high $\mathrm{Ca}^{2+}$ levels, TG2 can act as a crosslinking enzyme, catalyzing several post-translational modifications of target proteins. At low $\mathrm{Ca}^{2+}$ concentrations, TG2 may function as a G-protein or as a protein disulfide isomerase, thus contributing to the functionality of mitochondrial respiratory chain complexes [24-26]. Increased levels of TG2 are observed in several human pathologies including neurodegenerative diseases such as Alzheimer's, Huntington's, and Parkinson's diseases, as well as in chronic inflammatory conditions [24-26].

\section{Defective CFTR inhibits autophagy}

TG2 overactivation in the CF epithelial cells leads to functional sequestration of Beclin 1 (BECN1) [22], a protein essential for autophagy, a mechanism required for cell survival, and involved in the pathogenesis of several human diseases [27-30]. Autophagy is pivotal in promoting cellular clearance of protein aggregates and removal of ROS sources, such as damaged mitochondria and results in the lysosomal degradation of cytoplasmic organelles or cytosolic components after their sequestration in twomembraned vesicles (autophagosomes) [27-30]. BECN1 is a haploinsufficient tumor suppressor protein essential for autophagosome formation. BECN1 dissociates from Bcl-2 during stress conditions, such as starvation, thus promoting autophagy. Subsequently, BECN1 interacts with the class III phosphatidyl-inositol 3 kinase (PI3K), human vacuolar protein sorting (hVps)34, facilitating its activation. The ER-associated class III PI3K activity is crucial for the initiation of autophagosome formation [27-30].

We discovered that human and mouse CF airways exhibit a defect in autophagy, as indicated by reduced autophagosome formation, and the accumulation of sequestrosome 1 (SQSTM1) [22], a major autophagic substrate also known as p62 [31]. This occurs in spite of the normal expression of major autophagy genes [22]. A defective autophagic response to bacterial infection has also been reported in murine CF macrophages in which reduced autophagosome formation promotes the survival of Burkholderia cenocepacia, as well as the proinflammatory hypersecretion of IL-1 $\beta[32,33]$.

\section{Restoration of autophagy circumvents F508del-CFTR defect}

Genetic depletion of SQSTM1, transgene-enforced BECN1 overexpression, or addition of autophagy-stimulatory proteostasis regulators, such as cystamine (or its reduced form cysteamine), can increase the expression level of F508delCFTR protein and restore its function at the PM, either in CFTR homozygous CFBE41o- bronchial epithelial cell lines or in primary nasal epithelial cells freshly collected from CF patients bearing F508del-CFTR mutation, as well as in the lungs from $C f t r^{F 508 d e l}$ mice [34, 35]. As a consequence, restoration of autophagy reduces lung inflammation in Cftr ${ }^{\text {F508del }}$ mice. Notably, cysteamine has a long-lived action because this drug is able to allow F508del-CFTR to reside at the PM after rescue for $24 \mathrm{~h}$ beyond its washout $[34,35]$. These long-term effects of cysteamine are abrogated if CFTR function is simultaneously inhibited, or CFTR is depleted during washout [34, 35]. Importantly, the effects of cysteamine in reducing lung inflammation extend up to several days in vivo in $C f t r^{F 508 d e l}$ mice, unless autophagy is inhibited by 3-methyl-adenine (3-MA) after cysteamine withdrawal $[34,35]$. This suggests that the restoration of a proper autophagy flux can interrupt the CFTR-driven vicious cycle that derails the proteostasis network as, once stabilized at the epithelial surface, F508delCFTR can sustain its own PM residence and function.

\section{Proteostasis regulators prevent intestinal manifestations in CF mice}

In pigs and mice, the loss of the function mutations of Cftr causes a predominantly intestinal phenotype. Thus, 50 to $90 \%$ of $c f t r \mathrm{KO}$ and up to $40 \%$ of $C f t r{ }^{F 508 d e l}$ mice die within the first 4 weeks after birth, mostly due to intestinal obstruction, unless they are kept under a special diet that reduces the risk of obstruction [35]. We observed that oral treatment for 5 weeks with cysteamine significantly reduced mortality, improved weight gain, and increased the expression of functional CFTR protein at the intestinal level, at the same time, that it restored BECN1 protein expression to wild-type levels. Such a treatment is also capable of reducing lung inflammation [35].

\section{Proteostasis regulators increase F508del-CFTR stability}

Ensuring stability to the rescued F508del-CFTR mutant is a major concern in CFTR repairing therapies, as this mutant is rapidly dismissed from the PM after rescue and directed towards lysosomal degradation. Recent strategies aim at overcoming this issue through a combination of corrector and potentiator molecules, the 
latter aimed at enhancing channel activity after rescue. However, Cholon et al. and Veit et al., showed that the chronic administration of potentiators, such as Ivacaftor, is detrimental to F508del-CFTR stability, as it instead favors PM disposal of the mutant protein [36, 37]. Indeed, the mechanisms responsible for the premature F508del-CFTR removal from the PM after rescue are not fully understood. The rescued F508del-CFTR undergoes CHIP-mediated PM ubiquitination [38] and then is targeted by the ubiquitin-binding protein SQSTM1/p62, which accumulates at the PM as the consequence of disabled autophagy [34, 35]. The resulting complex composed by F508del-CFTR and SQSTM1/p62 is directed to the lysosomes for degradation [34, 35]. Moreover, defective PI3K complex III activity, due to functional sequestration of BECN1, impairs CFTR recycling, thus enhancing CFTR disposal.

Besides these autophagy-dependent mechanisms, other perturbations of the proteostasis network can influence F508del-CFTR stability at the PM. Peptide fragments released from proteolytically cleaved F508del-CFTR provoke an overactivation of a pleiotropic protein kinase (CK2), which in turn contributes to the fragmentation and poor stability of F508del-CFTR [39, 40]. The inhibition of overactive CK2 by specific inhibitors, such as CX-4945, or by means of natural compounds with known safety profile, as the over-the-counter flavonoid epigallocatechin gallate (EGCG), contributes to confer PM stability to F508del-CFTR mutant, thus prolonging the persistence of the mutant F508del-CFTR protein at the epithelial surface after rescue by cysteamine [35].

\section{Proteostasis regulators can restore F508del-CFTR function in CF patients}

Prompted by these pre-clinical results, we recently launched clinical pilot trials to evaluate the combination of cysteamine, already approved for patients with cystinosis (FDA number NDA020392) [41], and EGCG for the treatment of F508del-CFTR homozygous patients [30]. In this phase 2 trial, cysteamine plus EGCG restored autophagy and improved CFTR function while rescuing the expression of mature (band C) CFTR protein from the nasal epithelial cells in vivo. Notably, the functional rescue of F508delCFTR is coupled to, and correlated with, a decrease of chloride concentrations in sweat, as well as a reduction of the abundance of inflammatory cytokines in the sputum [35]. These findings indicate that such a combination treatment acts "on target". They also indicate that it is feasible to correct the F508delCFTR defect by manipulating the proteostasis network, thus rescuing the mutant CFTR protein and then normalizing its PM stability.

\section{Conclusions}

We believe that any CF treatment should aim at rescuing the expression and function of functional CFTR and hence target the true causes of the disease rather than its signs. Manipulation of proteostasis network offers new perspectives for the design of candidate drugs aimed at repairing the primary CFTR defect, well beyond the idea that such molecules must directly interact with mutant CFTR protein. The proteostasis network is unique to each cell type and tissues. Thus, finding appropriate proteostasis regulators implies a profound understanding of the pathogenic mechanisms operating within each peculiar cellular context. Based on our experience, we recommend a translational research approach that comprises studies on appropriate animal models and freshly collected primary cells from CF patients to develop a strong pre-clinical rationale before evaluating drugs in the clinic. Finally, we speculate that proteostasis modulators might represent an attractive approach to other human conformational diseases, including some neurodegenerative disorders, beyond CF.

\section{Conflict of interest}

All authors declare that they have no competing interests. LM, VR and GK are listed as inventors on a patent application (No. 13/895741) owned by the Noprofit Foundation European Institute for Research in Cystic Fibrosis, describing the use of cysteamine for the treatment of CF.

\section{Author details \\ ${ }^{1}$ European Institute for Research in Cystic Fibrosis, Division of Genetics and Cell Biology, San Raffaele Scientific Institute, Milan 20132, Italy. ${ }^{2}$ Regional Cystic Fibrosis Center, Pediatric Unit, Department of Translational Medical Sciences, Federico II University, Naples 80131, Italy. ${ }^{3}$ Equipe 11 labellisée par la Ligue contre le Cancer, Centre de Recherche des Cordeliers, Paris, France. ${ }^{4}$ Cell Biology and Metabolomics Platforms, GustaveRoussy Comprehensive Cancer Center, Villejuif, France. ${ }^{5}$ INSERM, U1138, Paris, France. ${ }^{6}$ Université Paris Descartes, Sorbonne Paris Cité, Paris, France. ${ }^{7}$ Université Pierre et Marie Curie, Paris, France. ${ }^{8}$ Pôle de Biologie, HôpitalEuropéen Georges Pompidou, AP-HP, Paris, France. ' Karolinska Institute, Department of Women's and Children's Health, Karolinska University Hospital, Stockholm, Sweden. ${ }^{10}$ SCDU of Pediatrics, Department of Health Sciences, University of Piemonte Orientale, Novara 28100, Italy.}

Received: 29 December 2015 Accepted: 7 March 2016

Published online: 14 March 2016

References

1. Ratjen F, Doring G (2003) Cystic fibrosis. Lancet 361:681-9

2. O'Sullivan BP, Freedman SD (2009) Cystic fibrosis. Lancet 373:1891-1904

3. Ratjen FA (2009) Cystic fibrosis: pathogenesis and future treatment strategies. Respir Care 54:595-605

4. Pittman JE, Ferkol TW (2015) The evolution of cystic fibrosis care. Chest 148: $533-42$

5. De Boeck K, Zolin A, Cuppens H, Olesen HV, Viviani L (2014) The relative frequency of CFTR mutation classes in European patients with cystic fibrosis. J Cyst Fibros 13:403-9

6. Amaral MD (2015) Novel personalized therapies for cystic fibrosis: treating the basic defect in all patients. J Intern Med 277:155-66

7. Ramsey BW, Davies J, McElvaney NG, Tullis E, Bell SC, Dřevínek P, Griese M, McKone EF, Wainwright CE, Konstan MW, Moss R, Ratjen F, Sermet-Gaudelus I, Rowe SM, Dong Q, Rodriguez S, Yen K, Ordoñez C, Elborn JS (2011) VX08770-102 Study Group. A CFTR potentiator in patients with cystic fibrosis and the G551D mutation. N Engl J Med 365(18):1663-72

8. Harrison MJ, Murphy DM, Plant BJ (2013) Ivacaftor in a G551D homozygote with cystic fibrosis. N Engl J Med 369:1280-2 
9. Van Goor F, Hadida S, Grootenhuis PD, Burton B, Stack JH, Straley KS, Decker CJ, Miller M, McCartney J, Olson ER (2011) Correction of the F508del-CFTR protein processing defect in vitro by the investigational drug VX-809. Proc Natl Acad Sci U S A 108:18843-8

10. Clancy J, Rowe SM, Accurso FJ, Aitken ML, Amin RS, Ashlock MA, Ballmann M, Boyle MP, Bronsveld I, Campbell PW (2012) Results of a phase lla study of VX-809, an investigational CFTR corrector compound, in subjects with cystic fibrosis homozygous fo the F508del-CFTR mutation. Thorax 67:12-8

11. Boyle AMP, Bell SC, Konstan MW, McColley SA, Rowe SM, Rietschel E, VX09-809-102 study group et al (2014) CFTR corrector (lumacaftor) and a CFTR potentiator (ivacaftor) for treatment of patients with cystic fibrosis who have a phe508del CFTR mutation: a phase 2 randomised controlled trial. Lancet Respir Med 2:527-538

12. Wainwright CE, Elborn JS, Ramsey BW, Marigowda G, Huang X, Cipolli M, TRAFFIC and TRANSPORT Study Groups et al (2015) Lumacaftor-Ivacaftor in patients with cystic fibrosis homozygous for Phe508del CFTR. N Engl J Med 373:220-231

13. Davis PB (2015) Another beginning for cystic fibrosis therapy. N Engl J Med 373:274-6

14. Jones AM, Barry PJ (2015) Lumacaftor/ivacaftor for patients homozygous for Phe508del-CFTR: should we curb our enthusiasm? Thorax 70:615-616

15. Balch WE, Roth DM, Hutt DM (2011) Emergent properties of proteostasis in managing cystic fibrosis. Cold Spring Harb Perspect Biol 3; doi: 10.1101/ cshperspect.a004499

16. Hutt DM, Herman D, Rodrigues AP, Noel S, Pilewski JM, Matteson J, Hoch B, Kellner W, Kelly JW, Schmidt A (2010) Reduced histone deacetylase 7 activity restores function to misfolded CFTR in cystic fibrosis. Nat Chem Biol 6:25-33

17. Pankow $S$, Bamberger $C$, Calzolari $D$, Martinez-Bartolomè $S$, Lavallée-Adam M, Balch WE, Yates III JR (2015) $\Delta$ F508 CFTR interactome remodelling promotes rescue of cystic fibrosis. Nature doi:10.1038/nature15729

18. Amaral MD, Balch WE (2015) Hallmarks of therapeutic management of the cystic fibrosis functional landscape. J Cyst Fibros 6:687-99

19. Villella VR, Esposito S, Bruscia EM, Vicinanza M, Cenci S, Guido S, PettoelloMantovani M, Carnuccio R, De Matteis MA, Luini A, Maiur MC, Raia V, Kroemer G, Maiuri L (2013) Disease-relevant proteostasis regulation of cystic fibrosis transmembrane conductance regulator. Cell Death Differ 20:1101-15

20. Maiuri L, Luciani A, Giardino I, Raia V, Villella VR, D'Apolito M, Pettoello-Mantovani M, Guido S, Ciacci C, Cimmino M, Cexus ON, Londei M, Quaratino S (2008) Tissue transglutaminase activation modulates inflammation in cystic fibrosis via PPARgamma down-regulation. J Immunol 180:7697-705

21. Luciani A, Villella VR, Vasaturo A, Giardino I, Raia V, Pettoello-Mantovani M, D'Apolito M, Guido S, Leal T, Quaratino S, Maiuri L (2009) SUMOylation of tissue transglutaminase as link between oxidative stress and inflammation. J Immunol 183:2775-84

22. Luciani A, Villella VR, Esposito S, Brunetti-Pierri N, Medina D, Settembre C, Gavina M, Pulze L, Giardino I, Pettoello-Mantovani M, D'Apolito M, Guido S, Masliah E, Spencer B, Quaratino S, Raia V, Ballabio A, Maiuri L (2010) Defective CFTR induces aggresome formation and lung inflammation in cystic fibrosis through ROS-mediated autophagy inhibition. Nat Cell Biol 12: 863-75

23. Villella VR, Esposito S, Bruscia EM, Maiuri MC, Raia V, Kroemer G, Maiuri L (2013) Targeting the intracellular environment in cystic fibrosis: restoring autophagy as a novel strategy to circumvent the CFTR defect. Front Pharmacol 4:1-8

24. Mastrobernardino PG, Piacentini M (2010) Type 2 transglutaminase in Huntington's disease a double-edged sword with clinical potential. J Intern Med 268:419-431

25. Piacentini M, D'Eletto M, Falasca L, Ferrace MG, Rodolfo C (2011) Transglutaminase 2 at the crossroad between cell death and survival. Adv Enzymol Relat Areas Mol Biol 78:197-246

26. Nurminskaya MV, Belkin AM (2012) Cellular functions of tissue transglutaminase. Int Rev Cell Mol Biol 294:1-97

27. Kroemer G, Mariño G, Levine B (2010) Autophagy and integrated stress response. Mol Cell 40:280-293

28. Mizushima N, Levine B, Cuervo AM, Klionsky DJ (2008) Autophagy fights disease through cellular self-digestion. Nature 28:1069-1075

29. Galluzzi L, Pietrocola F, Levine B, Kroemer G (2014) Metabolic control of autophagy. Cell 159:1263-76
30. Zhong Y, Wang QJ, Li X, Yan Y, Backer JM, Chait BT, Heintz N, Yue Z (2009) Distinct regulation of autophagic activity by Atg14L and Rubicon associated with Beclin1 phosphatidylinositol-3- kinase complex. Nat Cell Biol 11:468-476

31. Kirkin V, McEwan DG, Novak I, Dikic I (2009) A role for ubiquitinin selective autophagy. Mol Cell 34:259-269

32. Abdulrahman BA, Khwk AA, Akhter A, Caution K, Tazi M, Hassan H, Zhang Y, Rowland PD, Malhotra S, Aeffner F (2013) Depletion of the ubiquitinbinding adaptor molecule SQSTM1/p62 from macrophages harboring cftr DF508 mutation improves the delivery of Burkholderia cenocepacia to the autophagic machinery. J Biol Chem 288:2049-58

33. Assani K, Tazi MF, Amer AO, Kopp BT (2014) IFN-g stimulates autophagymediated clearance of Burkholderia cenocepacia in human cystic fibrosis macrophages. PLoS One 9, e96681

34. Luciani A, Villella VR, Esposito S, Gavina M, Russo I, Silano M, Guido S, Pettoello-Mantovani M, Carnuccio R, Scholte B, De Matteis A, Maiuri MC, Raia V, Luini A, Kroemer G, Maiuri L (2012) Targeting autophagy as a novel strategy for facilitating the therapeutic action of potentiators on DF508 cystic fibrosis transmembrane conductance regulator. Autophagy 8:1657-72

35. De Stefano D, Villella VR, Esposito S, Tosco A, Sepe A, De Gregorio F, Salvadori L, Grassia R, Leone CA, De Rosa G, Maiuri MC, Pettoello-Mantovani M, Guido S, Bossi A, Zolin A, Venerando A, Pinna LA, Mehta A, Bona G, Kroemer G, Maiuri L, Raia V (2014) Restoration of CFTR function in patients with cystic fibrosis carrying the F508del-CFTR mutation. Autophagy 10: 2053-74

36. Cholon DM, Quinney NL, Fulcher ML, Esther CR Jr, Das J, Dokholyan NV et al (2014) Potentiator ivacaftor abrogates pharmacological correction of $\Delta$ F508 CFTR in cystic fibrosis. Sci Transl Med 6:246ra96

37. Veit G, Avramescu RG, Perdomo D, Phuan PW, Bagdany M, Apaja PM et al (2014) Some gating potentiators, including VX-770, diminish $\Delta$ F508-CFTR functional expression. Sci Transl Med 6:246ra97

38. OkiyonedaT APM, Lukacs GL (2011) Protein quality control at the plasma membrane. Curr Opin Cell Biol 23:483-491

39. Venerando A, Cesaro L, Marin O, Donella-Deana A, Pinna LA (2014) A "SYDE" effect of hierarchical phosphorylation: possible relevance to the cystic fibrosis basic defect. Cell Mol Life Sci 71:2193-6

40. Cesaro L, Marin O, Venerando A, Donella-Deana A, Pinna LA (2013) Phosphorylation of cystic fibrosis transmembrane conductance regulator (CFTR) serine-511 by the combined action of tyrosine kinases and CK2: the implication of tyrosine-512 and phenylalanine-508. Amino Acids 45:1423-9

41. Gahl WA (2003) Early oral cysteamine therapy for nephropathic cystinosis. Eur J Pediatr 162:S38-S41

\section{Submit your manuscript to a SpringerOpen ${ }^{\circ}$ journal and benefit from:}

- Convenient online submission

- Rigorous peer review

- Immediate publication on acceptance

- Open access: articles freely available online

- High visibility within the field

- Retaining the copyright to your article

Submit your next manuscript at $\gg$ springeropen.com 